\title{
Heat Treatment of Gold Alloys
}

\section{THE MECHANISM OF DISORDER-ORDER HARDENING}

\author{
J. C. Chaston
}

Johnson Matthey \& Co Limited, London

\author{
By means of a relatively simple heat treatment operation the prop- \\ erties of certain gold alloys can be modified to give greatly increased \\ hardness and mechanical strength.
}

Gold, traditionally the softest of all metals, is remarkable as providing the basis for alloys which can be made intensely hard by the simplest of heat treatment operations - by cooling from a red heat.

The first known instance of any alloy so hardenable was in fact one which was predominantly of gold. This was developed as long ago as 1905 by the S. S. White Dental Manufacturing Company and placed on the American market in 1906. At the time, there was a demand for a hard wire which would not be softened locally when it was heated for hard soldering to small clasps for prosthetic purposes. It was found that a wire made from an alloy containing 64 per cent of gold, 12.5 per cent of platinum, 7 per cent of copper, and 16.5 per cent of silver met this requirement remarkably well.

The alloy is softened by quenching from about $600^{\circ} \mathrm{C}$, like most work-hardened non-ferrous materials, but hardens when cooled slowly - unlike almost any material then known. Later, it was found that the greatest hardening was secured by heating to about $400^{\circ} \mathrm{C}$ and cooling in the furnace. It may alternatively be quenched in water. And this treatment - originating with this alloy - is termed "tempering" by dentists to this day.

The curious behaviour of this alloy seems to have been accepted without much speculation as to its cause. The discovery of the age-hardening of the aluminium alloy Duralumin by Alfred Wilm a little later, in 1911, was much more widely publicised and sparked off quite extensive investigations. After Duralumin has been quenched from its annealing temperature into water, it hardens slowly when kept at room temperature, or much more rapidly by "tempering" at $100^{\circ} \mathrm{C}$ or higher. The mechanism of precipitation of a disperse phase from a supersaturated solid solution was established in a few years as an explanation of this behaviour.

The hardening of the gold alloy, however, was not so easily or so quickly explained. Curiously enough, the tempering treatment is effective in hardening the alloy whether or not it has been previously quenched; there is thus no question of the prior formation of a supersaturated solid solution. Further, the alloy does not over-age or soften when the reheating stage is prolonged, so there seems no disperse phase to agglomerate. It was not until the thirties that the concept of ordering in alloys was put forward, according to which the atoms in the alloy lattice take up preferred positions when the alloy is maintained in a certain zone of temperature. This change in itself will not necessarily produce a hardening effect.

Thus, in the first alloy in which ordering was recognised - the gold-copper alloy containing 25 atomic per cent or 50 weight per cent of gold - the effect is that the gold atoms go to the corners of the cubes in the crystal lattice and the copper atoms to the centres of the cube faces - but this involves no dimensional changes and has no influence on hardness. In the equiatomic gold-copper alloy that is used in the jewellery industry, containing 50 atomic per cent or 75 weight per cent of gold, however, ordering produces quite dramatic changes. On heating to 300 to $500^{\circ} \mathrm{C}$, the planes in the cubic cell arrange themselves into layers of all-gold and all-copper atoms. As a

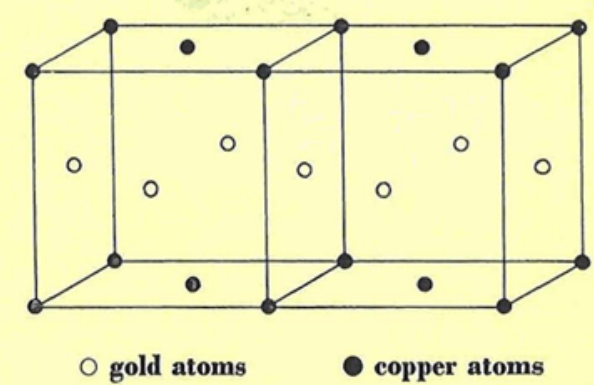

On heating the 50 atomic per cent gold-copper alloy to 300 to $500^{\circ} \mathrm{C}$ the planes in the facecentred cubic lattice arrange themselves into layers of all-gold and all-copper atoms. This gives rise to a small change in the lattice dimensions with a resultant marked increase in hardness and mechanical strength 
consequence, the height of the unit cell is decreased by about 5 per cent and the width and breadth are both increased by about 2.5 per cent. Such a change of shape - particularly when it is brought about at the lower temperatures in the zone of ordering - introduces very severe internal strains, with a consequent increase in hardness and strength.

This mechanism of atomic rearrangement at low temperatures, first established by studies on the simple binary alloys of gold and copper, has since been found to be a means of modifying the properties of many important industrial materials. Not only is it responsible - at least in part - for the effects of "tempering" the S. S. White dental alloy, but it is also involved in developing the extraordinary magnetic properties of cobalt-platinum hard magnetic alloys, in the high damping properties of the manganese-copper-nickel alloys, in the hardness of $\beta$ brasses, and even in the properties of many steels after heat treatment.

Today, ordering reactions are utilised to harden copper-containing gold alloys used for wipers in sliding electrical contacts. Appropriate heat treatment of a 62.5 per cent gold alloy containing copper and silver will, for example, produce a tensile strength of some 60 to 70 ton $/ \mathrm{in}^{2}$ with a Vickers hardness of about 275 . Ordering reactions are believed to occur in a range of alloys in the binary gold-vanadium, gold-zinc and gold-cadmium systems, though not a great deal is known of the precise mechanisms involved. It seems possible also that ordering may influence the performance of gold-palladium thermocouple alloys as well as of gold-nickel electrical contact alloys. There is undoubtedly scope for further research into these and other alloy systems.

\section{New Moving Coil Meter Suspensions}

\section{A HIGH STRENGTH GOLD ALLOY LIGAMENT}

The moving coil meter depends for its operation on the efficiency of its suspension. Hitherto, most of those developed for commercial use have incorporated suspension systems in which hardened steel pivots rotate in jewelled housings with delicate hairsprings to provide the restraining force. Such movements suffer from problems of wear, friction and susceptibility to damage from vibration or mechanical shock.

Some of the most delicate laboratory instruments of the galvanometer type use ligament suspensions in which the inherent twist of the ligament provides the restraining force but these have suffered from the disadvantage that the movements are free-hanging and therefore can be used only one way up.

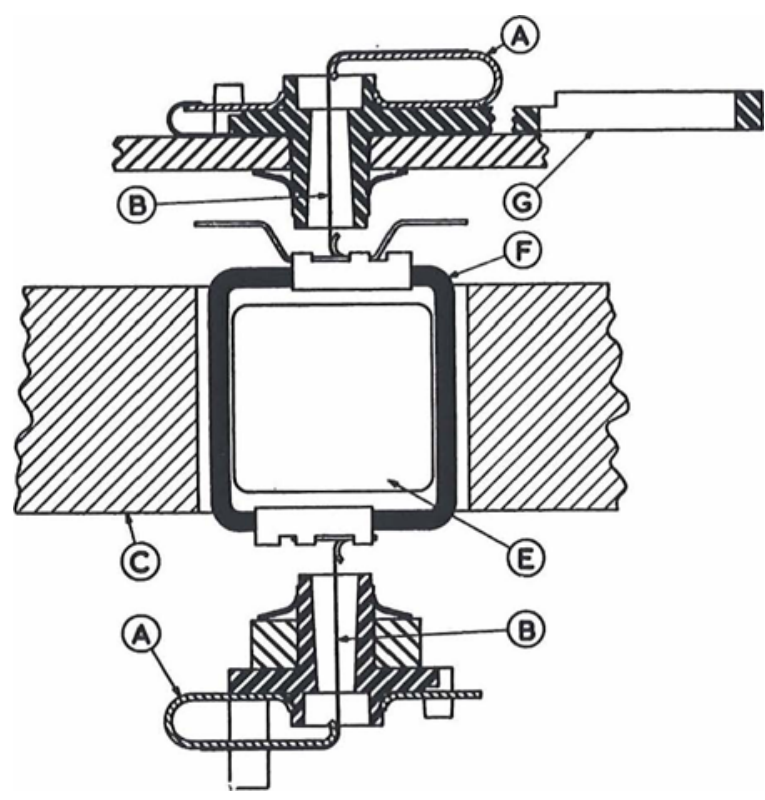

A few years ago Sifam Electrical Instrument Co., Torquay, commenced development of a commercial instrument based on the ligament principle. This operates with two ligaments held taut, one each side of the moving coil, and overcomes previous limitations of ligament suspension while possessing improved sensitivity and robustness.

Sifam refers to these movements as taut-band and they rely upon the ligament giving consistently the desired torque, remaining resilient, and having sufficient tensile strength, together with good electrical characteristics. A special stabilised silver-copper-gold alloy containing 62.5 per cent gold was selected to meet these requirements and it is, moreover, corrosionresistant. The new movement is claimed to be 25 times more sensitive than an equivalent pivoted movement. Hysteresis effects are minimised so that the pointer returns to within 0.25 per cent of true zero even after prolonged operation.

Successful development of these meters is leading Sifam to phase out production of pivoted movement meters altogether. Concentration on the new meter movement has reduced its cost to that of the pivoted movement and expansion of production is foreseen.

The construction of the taut-band movement developed by Sifam for moving coil meters. A - suspension spring, B - gold alloy ligament, C - magnet, $\mathbf{E}$ - core piece, F - coil, G - zero adjustment lever 